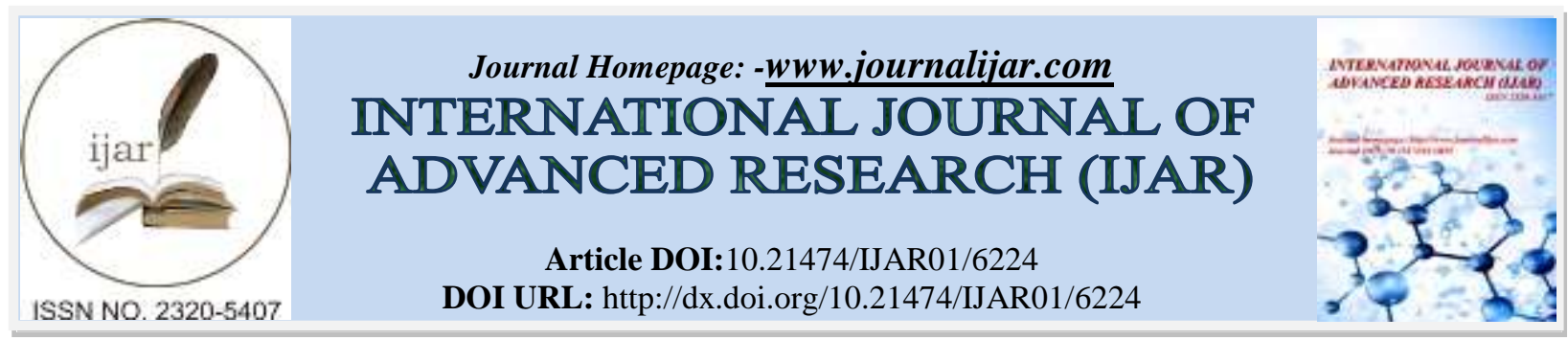

RESEARCH ARTICLE

\title{
EVALUATION OF ANGIOGENESIS IN ORAL EPITHELIAL DYSPLASIA AND ORAL SQUAMOUS CELL CARCINOMA: AN IMMUNOHISTOCHEMICAL COMPARISON BETWEEN ANTI-CD105 AND ANTI-CD31 ANTIBODIES.
}

\section{Amina Fouad Farag ${ }^{1}$, Rehab Fawzi Kasem ${ }^{2}$, Nahed Emad El-Din Abo-Azma ${ }^{3}$ and Mohsen Kazem Abd El- Latif $^{4}$.}

1. Assistant Lecturer of Oral Pathology, Faculty of Dentistry, October 6 University, Egypt.

2. Associate Professor of Oral and Maxillofacial Pathology, Faculty of Dentistry, Cairo University, Egypt.

3. Professor of Oral Pathology, Faculty of Dentistry, Tanta University, Egypt.

4. Professor of Oral and Maxillofacial Pathology, Faculty of Dentistry, Cairo University, Egypt.

\section{Manuscript Info}

Manuscript History

Received: 05 November 2017

Final Accepted: 07 December 2017

Published: January 2018

Key words:-

CD105, CD31, angiogenesis, MVD, OED, OSCC.

\begin{abstract}
Background and Aim: The tumor-induced angiogenesis is used as a reliable marker for tumor progression and metastasis. CD105 has been suggested as a marker with a greater specificity for tumor-induced angiogenesis since it is strongly up-regulated on proliferating endothelial cells of newly formed blood vessels while it shows no or only weak expression in normal pre-existing ones unlike CD31 which can not differentiate the newly formed tumoral vessels from normal pre-existing ones. This study was designed to measure the microvessel density (MVD) in oral epithelial dysplasia and oral squamous cell carcinoma using CD105 and CD31.

Methodology and Principal Findings: A total of 40 oral epithelial dysplasia and oral squamous cell carcinoma specimens were immunostained with CD105 and CD31. Then MVD was measured according to "hot spot method" in 3 high power microscopic fields at a magnification $\mathrm{x} 200$. The number of immunostained blood vessels increased from normal through dysplasia to carcinoma with a mean value of $1.5 \pm 0.5,3.2 \pm 1$ and $5.263 \pm 0.8$ for $\mathrm{CD} 105$ and $3.6 \pm 1.02$, $7.6 \pm 0.8$ and $9.77 \pm 1.75$ for CD31 respectively. CD31 showed positive immunoreaction in all blood vessels while CD105 revealed positive immunoreactivity only in the newly formed blood vessels while the native ones showed weak to negative immunoexpressions.

Conclusion: CD105 can be considered as a more specific marker for measuring the tumor-induced angiogenesis compared to the commonly used panendothelial marker CD31.
\end{abstract}

Copy Right, IJAR, 2018,. All rights reserved.

\section{Introduction:-}

Despite advances in the early cancer detection and its therapeutic interventions, most malignancies are still diagnosed and treated at very advanced stages with only limited range of therapeutic options and poor overall survival (Mariotto et al., 2011).

Corresponding Author:-AminaFouadFarag.

Address:-Assistant Lecturer of Oral Pathology, Faculty of Dentistry, October 6 University, Egypt. 
During the early stages of carcinogenesis, the ability of tumor to develop its own blood supply which is well known as the tumor-induced angiogenesis is considered a critical event separating the pre-invasive and dormant form of cancer from the invasive and metastatic phases of malignant growth and is considered one of most important hallmarks of cancer (Hanahan and Weinberg, 2011). It penetrates these tumor growths, supplies nutrients and oxygen and removes waste products by that crowded cell population for which the simple diffusion is no longer adequate (Buschmann and Schaper, 2000).

Quantification of tumor-induced angiogenesis can be made by counting microvasculars in a given area by immunohistochemical (IHC) staining which is so called the microvessel density (MVD) whose increase has been found to correlate with disease progression, poor prognosis and low survival rates (Chien et al., 2006).

By far, the most common antibodies used for microvessel staining are those against Factor VIII, CD31 and CD34. However, the accuracy of MVD assessment with these pan-endothelial markers may not be the highest possible since they cannot distinguish newly formed tumoral blood vessels (BVs) from normal pre-existed BVs which in turn rises the need for a marker that is strictly specific for tumor-induced angiogenesis (Martone et al., 2005 and Taskiran et al, 2006).

CD105 is a transforming growth factor beta (TGF- $\beta$ ) binding receptor preferentially expressed on endothelial cells (ECs) of all angiogenic tissues, including tumors, but weakly or not at all with those of normal tissues (Marioni et al., 2006) giving it the superiority as a marker with a greater specificity for tumor-induced angiogenesis. Moreover, its expression increased in the same time with the neo-angiogenic progression (Romani et al., 2006).

Although its exact mechanism of action is still under investigation, there is evidence on its role as a regulatory component of the receptor complex of the transforming growth factor- $\beta$ (TGF- $\beta$ ) which is a cytokine involved in tumor development and neoangiogenesis by regulating different cellular functions of ECs (Bernabeu et al., 2009).

A number of studies have shown the diagnostic and prognostic impact of CD105 in assisting tumor-induced angiogenesis in different solid malignancies such as skin cancer (Takahashi et al., 2001), breast carcinoma (Dales et al., 2003), prostatic cancer (Wikstrom et al., 2002), renal cell carcinoma (Costello et al., 2004), hepatocellular carcinoma (Ho et al., 2005) and ovarian carcinoma (Taskiran et al, 2006).

CD31 is an integral membrane glycoprotein belongs to immunoglobulin ( $\operatorname{Ig})$ gene superfamily that is involved in tumor-induced angiogenesis by promoting EC proliferation, migration and adhesion (Paddock et al., 2011).

CD31, like other panendothelial markers, aim at epitopes on ECs but not exclusively selective for them. They react with large and small BVs in addition to lymphatic, tumoral and inflammatory cells leading to their confused with microvessels during MVD measurement (Gee et al., 2003). This raises the need for a marker that is strictly specific for tumor-induced angiogenesis without any cross-reaction with the existing normal vessels or other populations in the background as the inflammatory cells (Duff et al., 2003).

Although CD105 were proven to be a powerful marker for the ongoing tumor-induced angiogenesis in such solid malignancies and has been accepted as a more accurate factor than other panendothelial markers such as CD31 and CD34 (Fonsatti and Maio, 2004 and Martone et al., 2005), only a few studies had investigated its expression in head and neck cancer.

The purpose of our study was to investigate the tumor-induced angiogenesis in oral epithelial dysplasia (OED) and oral squamous cell carcinoma (OSCC) using CD105 and CD31 and to correlate their expression with degree of dysplasia and grade of carcinoma.

\section{Materials and Methods:-}

Specimen Selection and Grouping: A total of 40 paraffin-embedded OED and OSCC specimens obtained from the archives of the Oral and Maxillofacial Pathology Department, Faculty of Dentistry, Cairo University and the Department of Surgical Pathology, National Cancer Institute, Cairo University were used in the present study. In addition to 8 specimens of normal appearing oral mucosa were taken from areas subjected to surgical procedure for purposes other than tumor removal to be employed as control group. 
Three serial $4 \mu \mathrm{m}$ thick sections were cut from each formalin-fixed, paraffin-embedded tissue block; one section was mounted on ordinary glass slide, for routine stain with $\mathrm{H} \& \mathrm{E}$ to be examined under ordinary light microscope in order to confirm previously made diagnosis of the selected specimens. The other two sections were mounted on positively charged slides for immunostained with anti-CD105 and anti-CD31 antibodies.

Based on the binary or two class system, the studied cases of OED were grouped according to the presence or absence of epithelial dysplasia into low risk showing questionable or mild dysplastic features in the basal and parabasal layers of epithelium and high risk showing moderate or severe dysplastic features extending to upper layers of epithelium (Kujan et al., 2006). On the other hand, the studied OSCC cases were classified according to WHO criteria into well, moderate and poorly differentiated where both well and moderately differentiated tumors are grouped together as low grade carcinomas and poorly differentiated and undifferentiated cases as high grade tumors (Pindborg et al., 1997).

Immunohistochemistry: was done using Strept-Avidin Biotin Complex $(\mathrm{ABC})$ universal kit (Neomarkers, Fermont CA, USA). As for antigen retrieval, we followed the ABC-IHC staining procedure as described by Wood \&Warnke (1981). The slides were divided into two groups where the first group was for mouse monoclonal anti-human CD105 antibody (clone: SN6h, isotype: IgG1/א and code no.: \#MS-1290-R7) and the second one for mouse monoclonal antihuman CD31 antibody (Clone: JC/70A, isotype: $\mathrm{IgG} 1 / \kappa$ and code no.: \#MS-353-R7). Both were ready to use primary antibodies supplied by Thermo Fisher Scientific Anatomical Pathology, Lab Vision Corporation, U.S.A.

Antibody expression and MVD quantification: The IHC evaluation of the stained sections was performed by two independent observers using the Leica Q550 image analyzer computer system. According to Hot spot MVD method as described by Weidner et al. (1991), the quantitative analysis of the tumor-induced angiogenesis was carried out for OSCC at three zones namely invading tumor front, inner tumor area and resection tumor margin (at least 3 optical fields far away from invading tumor front) and randomly for OED both in 3 high power microscopic fields at a magnification $\mathrm{x} 200$.

Any brown staining ECs or endothelial clusters that clearly separated from adjacent microvessels, tumor cells and other connective tissue elements were detected and considered as a single countable microvessel. However, vessels characterized by thick muscular walls or with lumen greater then $20 \mu \mathrm{m}$ in diameter were ignored and excluded from the counts. The Mean Hotspot MVD was determined by calculating average number of microvessels of the 3 microscopic fields then dividing this number by area of microscopic field in a standard measuring frame of 2927374 micrometer $^{2}(\mathrm{MV} / \mu \mathrm{m} 2)$ using a magnification of $\mathrm{x} 200$. This procedure was repeated for each tissue section and for both markers.

Statistical analysis: All the obtained data from the computer image analysis were then tabulated and statistically evaluated using using SPSS program (Statistical Package for Social Studies) version 19 software for windows and finally used for generation of a representative graphs. Paired Student t-test used to compare the mean values of MVD between two groups while ANOVA test used to compare mean between more than two groups. Both tests were considered to give a significant difference if $\mathrm{p}$ value was less than 0.005 .

\section{Results:-}

Immunohistochemical Findings and Hot Spot MVD Quantification:-

The IHC evaluation and quantification of hot spot CD105-MVD revealed positive CD105 immunoreactivity in the cytoplasm of ECs whether present singly or in clusters and in newly formed BVs while the native BVs with thick muscular walls and large lumina showed weak to negative CD105 immunoexpressions. On the other hand, all BVs whether newly formed or native showed strong positiveimmunoreactions to CD31antibodies in both normal and dysplastic specimens where reaction were detected in separate ECs, endothelial clusters and EC-lined microvessels whether thick or thin walled with large or small lumina. Thus, an increased number of BVs were stained with CD31 compared to CD105.

The control group revealed negative CD105 immunoexpression was detected in the BVs of 6 out of 8 cases while the remaining 2 cases showed weak positive immunoreactivity for CD105 in only few BVs. 
Positive CD105 immunoexpression were detected all cases of OED (low risk and high risk) and OSCC (low grade and high grade) except for 3 low risk OED cases where no positive immunostaining for CD105 was found in any of the BVs of the underlying connective tissue. However, positive immunoreactivity for CD31 was detected in all BVS of all OED and OSCC cases (figs. 1-4).

The number of immunostained BVs per microscopic field with its associated mean hot spot MVD for both markers is summarized in (table 1).

Table 1:- Number of BVs/microscopic field together with its associated Mean hot spot for CD105 and CD31-MVD:

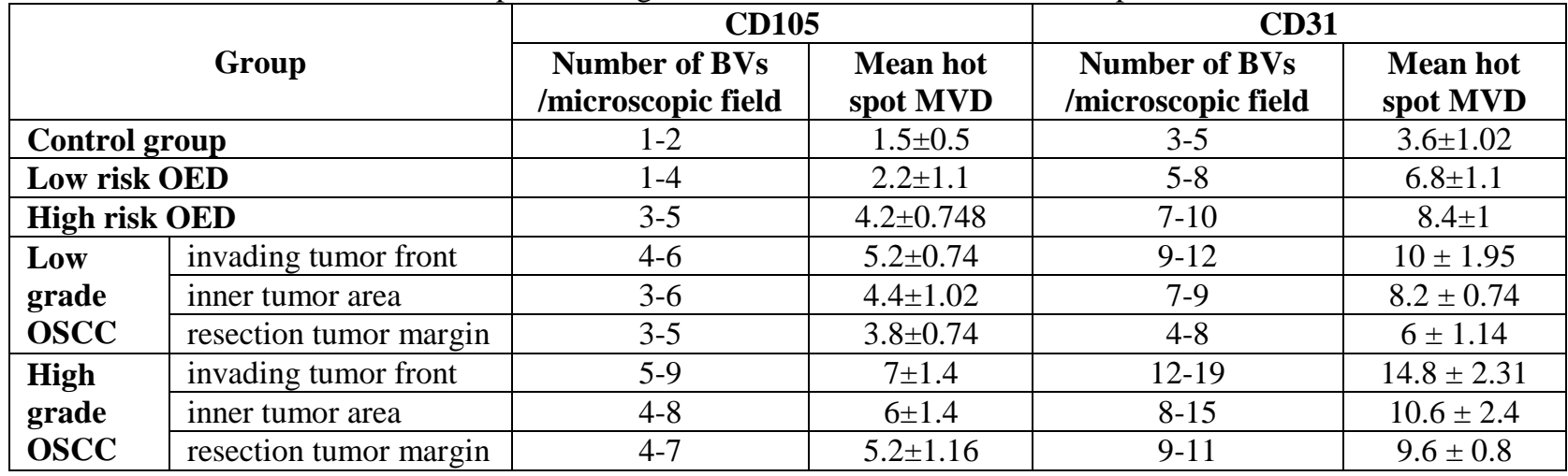

For both markers, the number of immunostained BVs increased from normal appearing mucosa through OED to OSCC cases and through the different degrees of OED and grades of OSCC where more BVs were found in cases with high risk OED compared to those with low risk OED and in those of high grade OSCC compared to that of low grade OSCC.

The highest number of immunostained BVs were recorded at the invading tumor front (peritumoral areas) followed by the inner tumor area (intratumoral areas) of all grades of OSCC compared to the resection tumor margin for both markers.

CD105 showed a completely negative immunoreaction in normal epithelial, neoplastic, inflammatory and mesechymal cells compared to positive immunoreaction for CD31 in some of them.

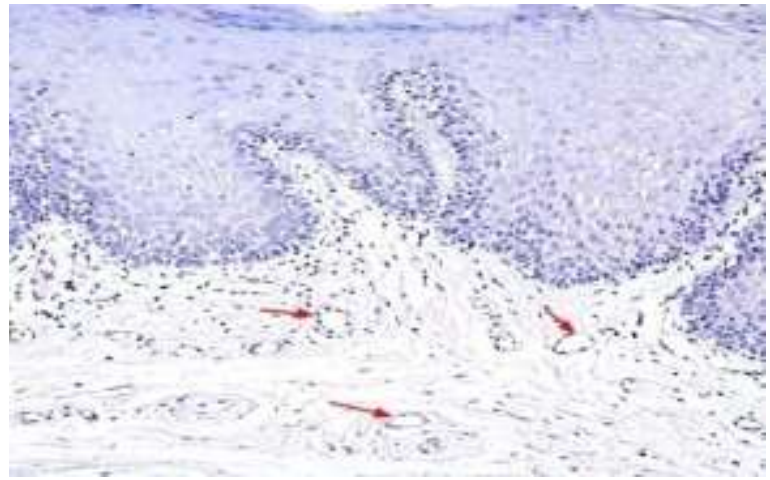

(1a)

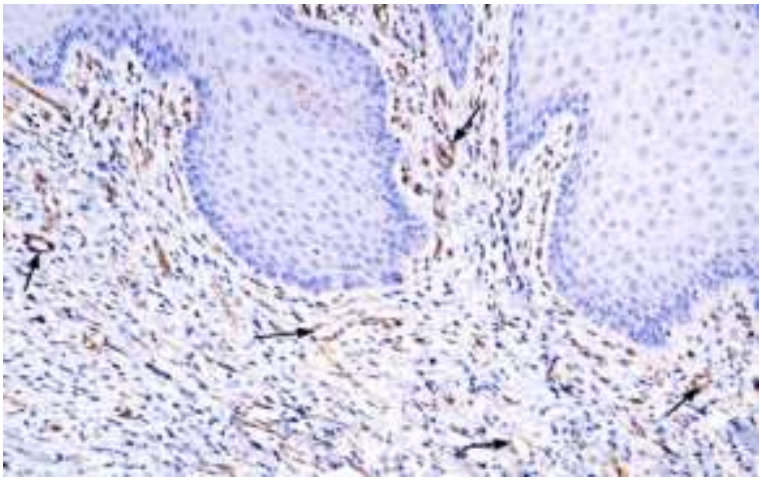

(1b) 


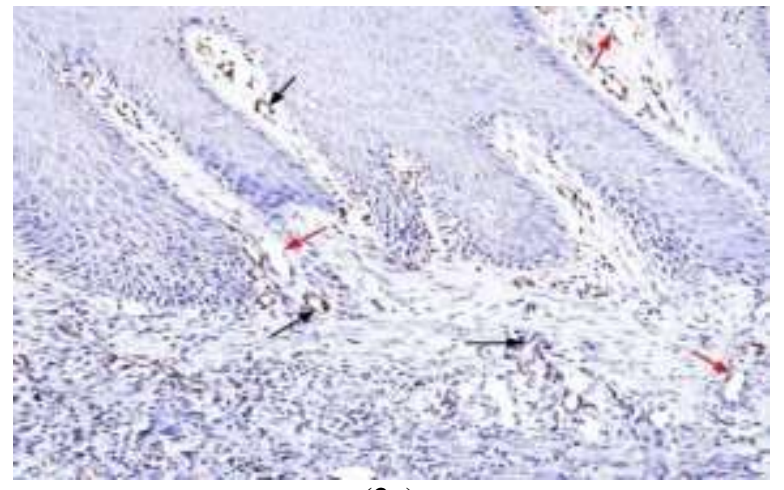

(2a)

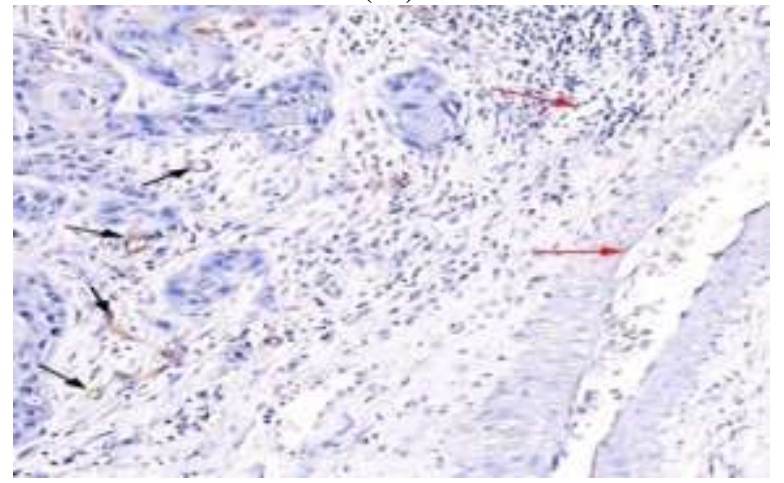

(3a)

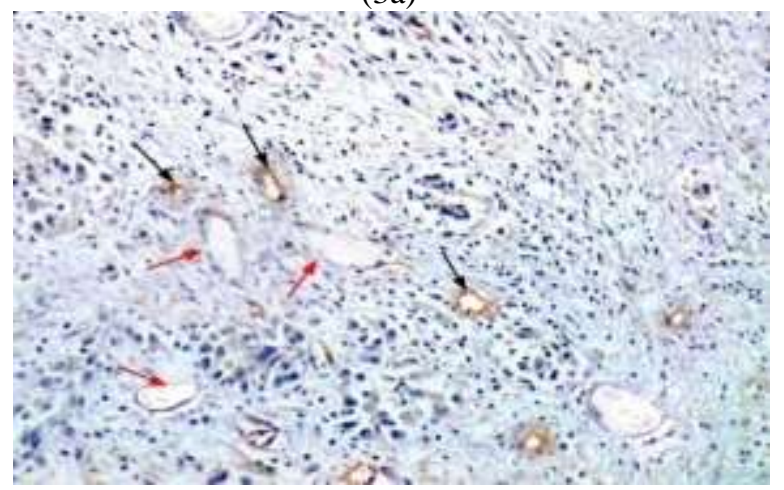

(4a)

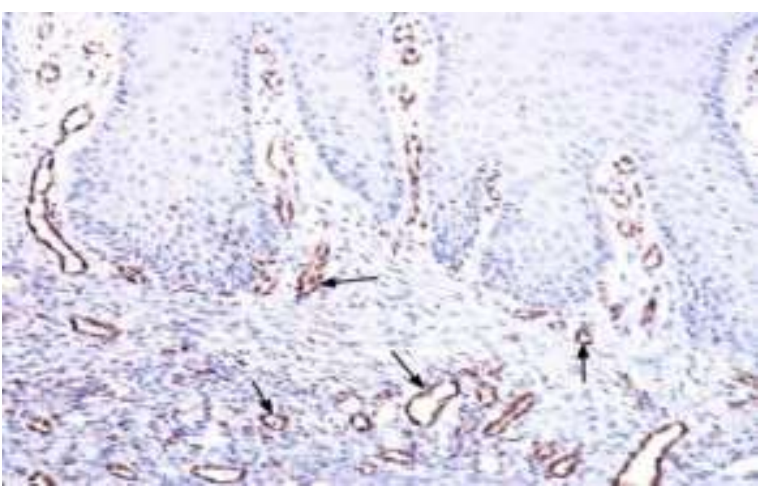

$(2 b)$

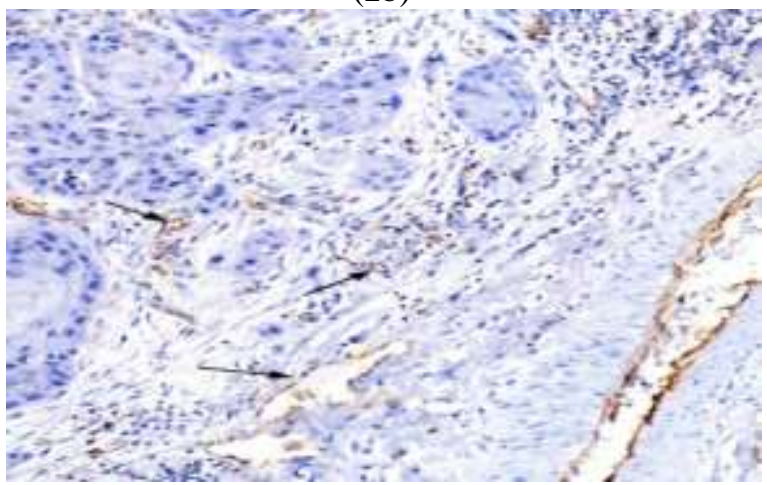

(3b)

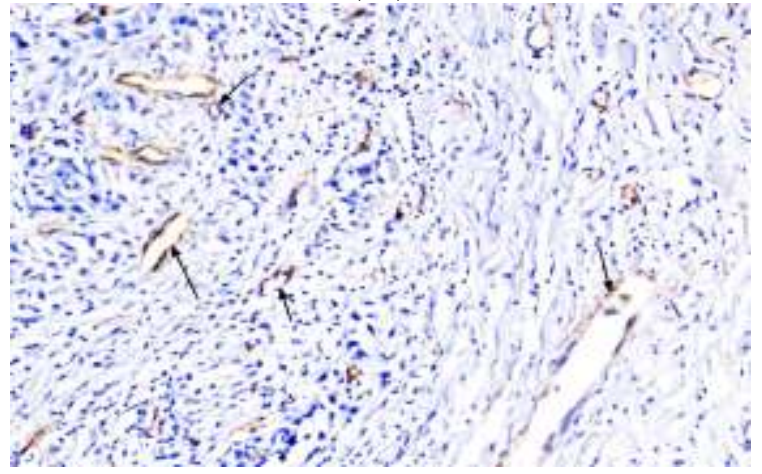

(4b)

Fig. 1:- Low risk OED cases stained with (a) CD105 and (b) CD31. Fig. 2. High risk OED stained with (a) CD105 and (b) CD31. Fig. 3. Low grade OSCC at invading tumor front (a) CD105 and (b) CD31. Fig. 4. High grade OSCC at invading tumor front (a) CD105 and (b) CD31. Black arrows show positively stained BVs while red show negatively stained ones (x200).

\section{Statistical Analyses:-}

The statistical analysis for mean hot spot MVD revealed a significant difference on comparing the low risk and high risk OED and a highly significant difference on comparing the low grade and high grade OSCC for both CD105 and CD31 (table 2, graph 1).

Difference in mean hot spot MVD between normal appearing, OED and OSCC cases revealed a highly significant difference in CD105-immunostained cases and a significant difference in CD31 immunostained cases. Thus, the hot spot MVD measured using CD31 was greater than that measured using CD105, (table 3, graph 2). 
Table 2:- Difference in Mean hot spot CD105-MVD and CD31-MVD using Paired Student t-test:

\begin{tabular}{|c|c|c|c|c|c|}
\hline \multirow{2}{*}{\multicolumn{3}{|c|}{ Group }} & \multicolumn{3}{|c|}{ Mean hot spot MVD } \\
\hline & & & $\mathbf{M} \pm \mathbf{S d}$ & t-Value & p-Value \\
\hline \multirow{4}{*}{ CD105 } & \multirow{2}{*}{ OED } & Low risk & $2.2 \pm 1.1$ & \multirow{2}{*}{3.2275} & \multirow{2}{*}{$0.0121 *$} \\
\hline & & High risk & $4.2 \pm 0.748$ & & \\
\hline & \multirow{2}{*}{ OSCC } & Low grade & $4.46 \pm 0.57$ & \multirow{2}{*}{6.6394} & \multirow{2}{*}{$0.001 * *$} \\
\hline & & High grade & $6 \pm 0.73$ & & \\
\hline \multirow{4}{*}{ CD31 } & \multirow{2}{*}{ OED } & Low risk & $6.8 \pm 1.1$ & \multirow{2}{*}{2.3164} & \multirow{2}{*}{$0.0492 *$} \\
\hline & & High risk & $8.4 \pm 1$ & & \\
\hline & \multirow{2}{*}{ OSCC } & Low grade & $8 \pm 1.63$ & \multirow{2}{*}{4.6974} & \multirow{2}{*}{$0.001 * *$} \\
\hline & & High grade & $11.52 \pm 2.3$ & & \\
\hline
\end{tabular}

$*=$ Statistically significant, $(\mathrm{p}<0.05)$

$* *=$ Highly statistically significant, $(\mathrm{p}<0.001)$.

Table 3:- Difference in Mean hot spot MVD between Normal appearing oral mucosa, OED and OSCC cases using ANOVA test:

\begin{tabular}{|c|c|c|c|c|}
\hline & \multirow{2}{*}{ Group } & \multicolumn{3}{|c|}{ Mean hot spot MVD } \\
\hline & & $\mathbf{M} \pm \mathbf{S d}$ & f-Value & p-Value \\
\hline \multirow{3}{*}{ CD105 } & Controlgroup & $1.5 \pm 0.5$ & \multirow{3}{*}{12.19298} & \multirow{3}{*}{$0.000067 * *$} \\
\hline & OED & $3.2 \pm 1$ & & \\
\hline & OSCC & $5.263 \pm 0.8$ & & \\
\hline \multirow{3}{*}{ CD31 } & Control & $3.6 \pm 1.02$ & \multirow{3}{*}{20.59605} & \multirow{3}{*}{$0.00001 * *$} \\
\hline & OED & $7.6 \pm 0.8$ & & \\
\hline & OSCC & $9.77 \pm 1.75$ & & \\
\hline
\end{tabular}

$* *=$ Highly statistically significant, $(\mathrm{p}<0.001)$.

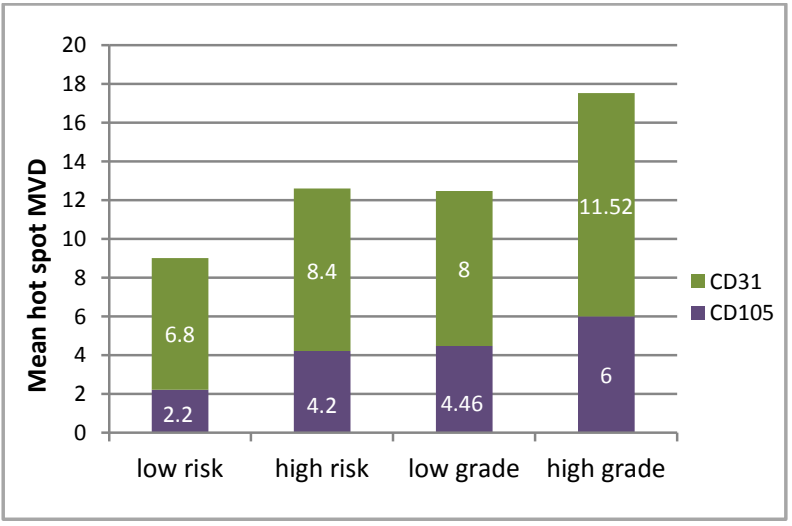

Graph 1:- Bar chart illustrating Mean hot spot MVD of CD105 and CD31 in OED (low \& high risk) and OSCC (low \& high grade) cases.

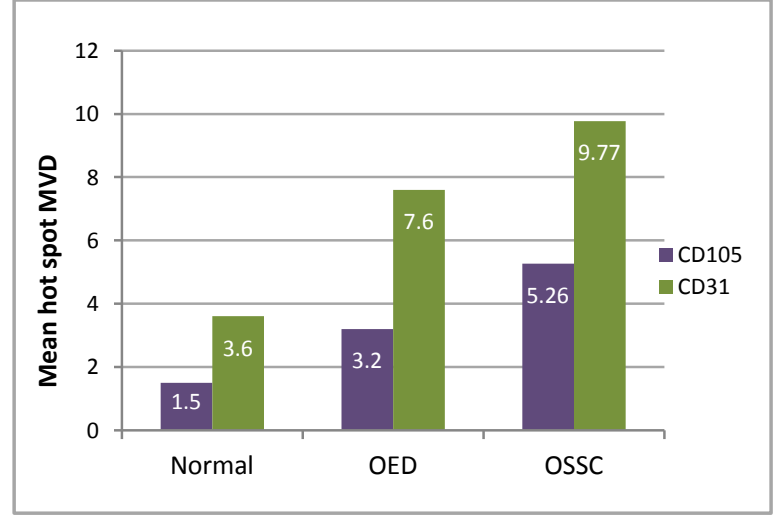

Graph 2:- Bar chart illustrating Mean hot spot CD105 and CD31-MVD in normal appearing, OED and OSCC cases.

\section{Discussion:-}

The identification of new diagnostic and prognostic markers for the early tumor detection, progression and metastasis has been the focus of most cancer studies. Such markers could serve as targets for anti-neoplastic therapies and ultimately reduce the morbidity and mortality associated with cancer (Seki et al., 2011).

The fact that no solid tumors can grow up more than $2-3 \mathrm{~mm}$ in diameter without the induction of its own blood supply (Folkman, 2000) strongly suggested tumor-induced angiogenesis as a significant indicator for cancer progression that correlates positively with disease stage, the likelihood of metastases and recurrence (Thomas et al., 2007). 
Studies on the available panendothelial markers as CD31 had shown their low sensitivity and specificity in evaluation of the tumor-induced angiogenesis and even yielded conflicting and contradictory results. The CD105 seems to react specifically with actively proliferating ECs and to be associated with the increase in MVD occurring during transition from normal oral mucosa through different grades of dysplasia to invasive carcinoma (Siar et al., 2009).

In the current work, the negative CD105 immunoexpression in the control group is explained on the basis that only actively proliferating ECs can secreted pro-angiogenic factors such as CD105 which exert their angiogenic effects by binding to and modulating the TGF- $\beta 1$ pathway leading downstream events of angiogenic cascade while the resting ECs of normal vasculature do not produce any pro-angiogenic factors unless become activated by inflammation, healing of normal tissues or multistep carcinogenesis (Bellone et al., 2007).

However, the CD31 showed positive immunoexpression in all the control group cases, but the number of positively stained BVs were lower than that recorded in neoplastic cases. Basilio de Oliveira \& Pannain (2015) attributed this to the inability of CD31 to distinguish newly formed tumoral BVs from normal pre-existed ones in both neoplastic and non-neoplastic tissues and the fact that they are better expressed in larger vessels than in microvessels.

In 4 out of the 7 cases of low risk OED, positive CD105 immunoreactivity was reported in only a small number of BVs. The supportive role of cellular components of the ECM such as tumor associated fibroblasts and inflammatory cells during the multistep carcinogenesis may explain this finding where the initiation of genetic mutations in the epithelial cells lead to activation of the ECM which in turn express growth factors capable of supporting the growth, proliferation and survival of cancerous cells by many mechanisms including the induction of angiogenesis (Basnaker et al., 2014).

Therefore, the negative CD105 immunoexpression reported in remaining 3 cases of low risk OED may be attributed to fact that tumor cells did not stimulate the cellular components of the ECM to activate the tumor-induced angiogenesis which may in part explain the susceptibility of some of the low risk OED cases for acquiring further malignant changes and progress to malignancy while others may never (Sperandio et al., 2013).

Positive immunoexpression for both CD105 and CD31 was detected in all the high risk OED and all grades of OSCC. However, CD105 was exclusively expressed on actively proliferating ECs of newly formed BVs but not on the normal pre-existing ones while CD31 was found to stain all BVs indiscriminately whether newly formed small or native large ones. Such finding is related to the selective staining of CD105 to actively proliferating ECs (Nagatsuka et al., 2005) on the contrary to CD31 which is not selective for epitopes on ECs but also aimed at those on inflammatory, mesechymal, neoplastic and non-neoplastic epithelial cells leading to their confused with microvessels (Taskiran et al, 2006).

Both CD105- and CD31-MVD increased from the low risk OED cases to high risk ones and from low to high grade OSCC cases where higher MVD was recorded in the OSCC compared to OED cases. Dassoulas et al. (2010), Romero et al. (2011) and Lertkiatmongkol et al. (2016) agreed to explain this finding based on the Folkman's concept that tumor growth and progression is angiogenic dependent in order to meet the increased metabolic demand of neoplastic cells as the tumor progress from normal mucosa through dysplasia to carcinoma and through different degrees of dysplasia and grades of carcinoma and in turn increased release of pro-angiogenic factors by the tumor cells themselves and their associated ECM to stimulate tumor-induced angiogenesis in order to compensate for that demand.

The highest MVD for both markers were detected in OSSC where the high grade were higher than low grade cases with the invading tumor front showed the greatest counts in all the three measuring zones. In CD105 cases, the presence of actively proliferating ECs or immature neovessels with strong remodeling activity at the invading tumor front and inner tumor area compared to the low remodeling activity of the BVs at resection tumor edge explains such finding (Tadbir et al., 2014 and Nair et al., 2016). Similarly, de Oliveira et al. (2013) and Fernández et al. (2017) attributed this to the ability of the CD31 to alter the cytoskeleton to form filopodial protrusions, both by dephosphorylating focal adhesion kinase as well as by altering the activity of the small G-protein, Ras homolog gene family, member A leading to endothelial cell proliferation, migration and adhesion and in turn activation of tumorinduced angiogenesis. 
Although Mărgăritescu et al. (2008) had also reported that expression of CD105 in the neoplastic tissues was significantly higher than in normal healthy mucosa and declined as they moved away from the invading tumor front respectively, they did not found any significant correlation between of MVD with the tumor stage or the grade of differentiation on the contrary to our results where CD105-MVD were found to significantly correlate with the degree of OED and highly significantly correlate with grade of OSCC.

Such controversial and discrepancies in the results is properly owned to the methodological variations between these studies, inter-observer variability in the identification and selection of the hot spots or may be due to differences in IHC protocols and counting procedure (Li et al., 2009).

Analysis of mean MVD in both OED and OSSC cases stained with CD31 antibodies revealed much higher values than those stained with CD105 antibodies. This is explained by the fact that CD31 stained both small tumoral vessels with active endothelial cells and large native vessels with resting endothelial cells on the contrary to CD105 who stained only active endothelial cells but not resting ones (Mitselou et al., 2016).

\section{Conclusions:-}

In conclusion, both markers in present work showed that MVD increased with disease progression from the low risk to the high risk OED cases then from the low grade to the high grade OSCC cases. However, the assessment of tumor-induced angiogenesis using panendothelial markers such as CD31 was apparently unfit as such markers were not selective for newly formed active tumoral BVs but they were even better expressed on the normal resting ones and show cross reactivity with other cells in the tumor background especially the inflammatory cells which may lead to wrong MVD counts due their confused with microvessels especially in the inflamed cases. On the contrary, CD105 as a marker with a greater specificity for tumor-induced angiogenesis had provided more accurate measures and easier measuring protocol with no discrepancies.

\section{Acknowledgement:-}

We would like to thank all the members of the Research Centers in Faculty of Dentistry, Cairo University and in the Faculty of Dentistry, Tanta University for the facilities they provided to the authors during the course of this work.

\section{Conflict Of Interest:-}

The authors declare that they have no conflict of interest.

\section{References:-}

1. Basilio de Oliveira, R. and Pannain, V. (2015): Prognostic angiogenic markers (Endoglin, VEGF, CD31) and tumor cell proliferation (Ki67) for gastrointestinal stromal tumors. World J. Gastroenterol., 21(22):6924-6930.

2. Basnaker, M.; Sr, S. and Bnvs, S., 2014. Expression of endoglin (CD105) and microvessel density in oral dysplasia and squamous cell carcinoma. J. Clin. Diagn. Res., 8(9):ZC91-ZC94.

3. Bellone, G.; Solerio, D.; Chiusa, L.; Brondino, G.; Carbone, A.; Prati, A.; Scirelli, T.; Camandona, M.; Palestro, G. and Dei Poli, M. (2007): Transforming growth factor- $\beta$ binding receptor endoglin (CD105) expression in esophageal cancer and in adjacent nontumorous esophagus as prognostic predictor of recurrence. Ann. Surg. Oncol., 14(11):3232-3242.

4. Bernabeu, C.; LópezNovoa, J. and Quintanilla, M. (2009): An emerging role of TGF- $\beta$ co-receptors in cancer. Biochim. Biophys. Acta., 1792(10):954-973.

5. Buschmann, I. and Schaper, W. (2000): The pathophysiology of the collateral circulation (arteriogenesis). J. Pathol., 190(3):338-342.

6. Chien, C.; Su, C.; Hwang, C.; Chuang, H.; Hsiao, Y.; Wu, S. and Huang, C. (2006): Clinicopathologic significance of CD105 expression in squamous cell carcinoma of the hypopharynx. Head Neck, 28(5):441-446.

7. Costello, B.; Li, C.; Duff, S.; Butterworth, D.; Khan, A.; Perkins, M.; Owens, S.; Al Mowallad, A.; O'Dwyer, S. and Kumar, S. (2004): Perfusion of 99Tcm-labeled CD105 Mab into kidneys from patients with renal carcinoma suggests that CD105 is a promising vascular target. Int. J. Cancer, 109(3):436-441.

8. Dales, J.; Garcia, S.; Bonnier, P.; Andrac Meyer, L.; Ramuz, O. Lavaut, M.; Allasia, C. and Charpin C. (2003): CD105 expression is a marker of high metastatic riskand poor outcome in breast carcinomas: Correlations between immunohistochemical analysis and long-term follow-up in a series of 929 patients. Am. J. Clin. Pathol., 119(3):374-380. 
9. Dassoulas, K.; Gazouli, M.; Theodoropoulos, G.; Christoni, Z.; Rizos, S.; ZisiSerbetzoglou, A.; Glava, C.; Karantanos, T.; Klonaris, C. and Karakitsos, P. (2010): Vascular endothelial growth factor and endoglin expression in colorectal cancer. J. Cancer Res. Clin. Oncol., 136(5):703-708.

10. de Oliveira, M.; Pereira Gomes, E.; Pereira, C.; de Souza, L.; Barros, L.; Mendes, D.; Guimarães, A. and De Paula, A. (2013): Prognostic value of microvessel density and p53 expression on the locoregional metastasis and survival of the patients with head and neck squamous cell carcinoma. Appl. Immunohistochem. Mol. Morphol., 21(5):444-451.

11. Duff S., Li C., Garland J. and Kumar S. (2003): CD105 is important for angiogenesis: Evidence and potential applications. FASEB J; 17:984-92.

12. Fernández, A.; Fernández, J.; Marshall, M.; Martínez, R.; Niklander, S. and Haidar, Z. (2017): Difference in EGFR expression and mean vascular density in normal oral mucosa, oral epithelial dysplasia and oral squamous cell carcinoma. J. Oral Res., 6(2):39-45.

13. Folkman, J. (2000): Incipient angiogenesis. J Nati Cancer Inst., 92(2), 94-5.

14. Fonsatti, E. and Maio, M. (2004): Highlights on endoglin (CD105): From basic findings towards clinical applications in human cancer. J. Transl. Med., 2(1):18.

15. Gee, M., Procopio, W., Makonnen, S., Feldman, M., Yeilding, N. and Lee, W. (2003): Tumor vesseldevelopment and maturation impose limits on theeffectiveness of anti-vascular therapy. Am. J. Pathol., 162(1):183-93.

16. Hanahan, D. and Weinberg, R.. (2011): Hallmarks of Cancer: The Next Generation. Cell, 144(5):646-674.

17. Ho, J.; Poon, R.; Sun, C.; Xue, W. and Fan, S. (2005): Clinicopathological and prognostic implications of endoglin (CD105) expression in hepatocellular carcinoma and its adjacent non-tumorous liver. World J. Gastroenterol., 11(2):176-181.

18. Kujan, O.; Oliver, R.; Khattab, A.; Roberts, S.; Thakker, N. and Sloan, P. (2006): Evaluation of a new binary system of grading oral epithelial dysplasia for prediction of malignant transformation. Oral Oncol., 42(10):987993.

19. Lertkiatmongkol, P.; Liaoa, D.; Meib, H.; Hub, Y. and Newmana, P. (2016): Endothelial functions of platelet/endothelial cell adhesion molecule-1 (CD31). Curr. Opin. Hematol., 23(3):253-259.

20. Li, S.; Hung, P.; Chou1, K.; Hsieh, S. and Shieh, Y. (2009): Tumor Angiogenesis in Oral Squamous Cell Carcinomas:The Significance of Endothelial Markers and Hotspot Selection. J. Med. Sci., 29(2):67-74.

21. Mărgăritescu, C.; Simionescu, C.; Mogoantă, L.; Badea, P.; Pirici, D.; Stepan, A. and Ciurea, R. (2008): Endoglin (CD105) and microvessel density in oral squamous cell carcinoma. Rom. J. Morphol. Embryol., 49(3):321-326.

22. Marioni, G.; Marino, F.; Giacomelli, L.; Staffieri, C ;Mariuzzi, M.; Violino, E. and De Filippis, C. (2006): Endoglin expression is associated with poor oncologic outcome in oral and oropharyngeal carcinoma. Acta. Otolaryngol., 126(6):633-639.

23. Mariotto, A.; Yabroff, K.; Shao, Y.; Feuer, E. and Brown, M. (2011): Projections of the cost of cancer care in the United States: 2010-2020. J. Nati. Cancer Inst., 103(2):117-128.

24. Martone, T.; Rosso, P.; Albera, R.; Migliaretti, G.; Fraire, F.; Pignataro, L.; Pruneri, G.; Bellone, G. and Cortesina, G. (2005): Prognostic relevance of CD105 microvessel density in HNSCC patient outcome. Oral Oncol., 41(2):147-155.

25. Mitselou, A.; Galani, V.; Skoufi, U.; Arvanitis, D.; Lampri, E and Ioachim, E. (2016): Syndecan-1, EpithelialMesenchymal Transition Markers (E-cadherin/ $\beta$-catenin) and Neoangiogenesis-related Proteins (PCAM-1 and Endoglin) in Colorectal Cancer. Anticancer Res., 36(5):2271-2280.

26. Nagatsuka, H.; Hibi, K.; Gunduz, M.;Gunduz, M.; Tsujigiwa, H.; Tamamura, R.; Sugahara, T.; Sasaki, A. and Nagai, N. (2005): Various immunostaining patterns of CD31, CD34 and endoglin and their relationship with lymph node metastasis in oral squamous cell carcinomas. J. Oral Pathol. Med., 34(2):70-76.

27. Nair, S.; Nayak, R.; Bhat, K.; Kotrashetti, V. and Babji, D. (2016): Immunohistochemical expression of CD105 and tgf- $\beta 1$ in oral squamous cell carcinoma and adjacent apparently normal oral mucosa and its correlation with clinicopathologic features. Appl. Immunohistochem. Mol. Morphol., 24(1):35-41.

28. Paddock, C., Lytle, B., Peterson, F., Holyst, T., Newman, P., Volkman, B., et al. (2011): Residues within a lipid-associated segment of the PECAM-1 cytoplasmic domain are susceptible to inducible, sequential phosphorylation. Blood; 117:6012-23.

29. Pindborg, J.; Reichart, P.; Smith, C. and van der Wall, I. (1997): Carcinomas. In: Altini, M.; Anneroth, G.; Bhonsle, R. et al. (eds) Histological Typing of Cancer and Precancer of the Oral Mucosa, $2^{\text {nd }}$ Edition. Berlin,Germany, Springer-Verlag Berlin and Heidelberg GmbH \& Co. KG, pp:11-16. 
30. Romani, A.; Borghetti, A.; Del Rio, P.; Sianesi, M. and Soliani, P. (2006): The risk of developing metastatic disease in colorectal cancer is related to CD105-positive vessel count. J. Surg. Oncol., 93(6):446-455.

31. Romero, D.; O'Neill, C.; Terzic, A.; Contois, L.; Young, K.; Conley, B.; Bergan, R.; Brooks, P. and Vary, C. (2011): Endoglin regulates cancer-stromal cell interactions in prostate tumors. Cancer Res., 71(10):3482-3493.

32. Seki, S., Mutsunori, F., Fujiwara, M., Matsuura, M., Fujita, S., Ikeda, H., et al. (2011): Prediction of outcome of patients with oral squamous cell carcinoma using vascular invasion and the strongly positive expression of vascular endothelial growth factors. Oral Oncol.; 47(7):588-93.

33. Siar, C.; Oo, V.; Nagatsuka, H.; Nakano, K.; Ng, K. and Kawakami, T. (2009): Angiogenic squamous dysplasia-like phenomenon in oral epithelial precursor lesions. Eur. J. Med. Res.,14(7):315-319.

34. Sperandio, M. ; Brown, A.; Lock, C. ; Morgan, P. ; Coupland, V.; Madden, P. ; Warnakulasuriya, S.; Møller, H and Odell, E. (2013): Predictive value of dysplasia grading and dnaploidy in malignant transformation of oral potentially malignant disorders. Cancer Prev. Res., 6(8):822-831.

35. Tadbir, A.; Ashraf, M. and Moradi, M. (2014): Clinicopathological Significance of CD105 Expression in Squamous Cell Carcinoma of the Oral Cavity. MEJC, 5(1):7-12.

36. Takahashi, N.; Haba, A.; Matsuno, F. and Seon, B. (2001): Antiangiogenic therapy of established tumors in human skin/severe combined immunodeficiency mouse chimeras by anti-endoglin (CD105) monoclonal antibodies and synergy between anti-endoglin antibody and cyclophosphamide. Cancer Res., 61(21):7846-7854.

37. Taskiran, C.; Erdem, O.; Onan, A.; Arisoy, O.; Acar, A.; Vural, C.; Erdem, M.; Ataoglu, O. and Guner, H. (2006): The prognostic value of endoglin (CD105) expression in ovarian carcinoma. Int. J. Gynecol. Cancer, 16(5):1789-1793.

38. Thomas, B., Eyries, M., Montagne, K., Martin, S., Agrapart, M., Simerman-François, R. et al. (2007): Altered endothelial gene expression associated with hereditary haemorrhagic telangiectasia. Eur. J. Clin. Invest., 37:580-8.

39. Weidner, N.; Semple, J.; Welch, W. and Folkman, J. (1991): Tumor angiogenesis and metastasis-correlation in invasive breast carcinoma. N. Engl. J. Med., 324(1):1-8.

40. Wikstrom, P.; Lissbrant, I.; Stattin, P.; Egevad, L. and Bergh, A. (2002): Endoglin (CD105) is expressed on immature blood vessels and is a marker for survival in prostate cancer. Prostate, 51(4):268-275.

41. Wood, G. and Warnke, R. (1981): Suppression of endogenous avidin-binding activity in tissues and its relevance to biotin-avidin detection system. J. Histochem. Cytochem., 29(10):1196-1204. 